\title{
Quantum transport in zigzag graphene nanoribbon decorated with a phenanthrene molecule
}

\section{Bo Jiang}

Kunming University of Science and Technology. School of Physics and Astronomy, Yunnan University.

\section{Qing-Yuan Chen}

Kunming University

Jinlong Luo

Kunming University

Yifen Zhao

Kunming University

GuoJun Jin

Kunming University

Hai Yang ( $\nabla$ kmyangh@263.net )

Kunming University

\section{Research Article}

Keywords: Quantum transport, Molecular device, Graphene nanoribbon, First principles calculation

Posted Date: July 1st, 2021

DOl: https://doi.org/10.21203/rs.3.rs-164631/v1

License: (c) (1) This work is licensed under a Creative Commons Attribution 4.0 International License.

Read Full License 


\title{
Quantum transport in zigzag graphene nanoribbon decorated with a phenanthrene molecule
}

\author{
Bo Jiang ${ }^{1,2}$ • Qing-Yuan Chen ${ }^{1}$ • Jin-Long \\ Luo $^{1}$. Yi-Fen Zhao ${ }^{1}$. Guojun $\mathrm{Jin}^{1,3,{ }^{\dagger}}$. \\ Hai Yang ${ }^{1, *}$
}

Received: date / Accepted: date

\begin{abstract}
By using the first principles calculations which combine density functional theory and nonequilibrium Green's function, we investigate the nanoscopic quantum transport in three hybrid structures consisting of a phenanthrene (PHE) molecule and a zigzag graphene nanoribbon (ZGNR). It is found that after decorated with the PHE molecule, the ZGNR with the odd (even) zigzag carbon chains shows the conductance reduction (enhancement), respectively. With the increase of the number of carbon chains, this odd-even difference will disappear. Moreover, negative differential resistance behavior can also be found in the hybrid structures consisting of the PHE molecule and the antisymmetric ZGNR. Finally, the differential conductance, transmission spectra, and molecular projection self-consistent Hamiltonian are used to explain the physical mechanism clearly. Accordingly, the proposed structures could have broad applications in the design of molecular nanodevices.
\end{abstract}

Keywords Quantum transport · Molecular device · Graphene nanoribbon · First principles calculation

\section{Introduction}

Molecular devices are ultra-miniature constructions. As early as 1974, Aviram and Ratner firstly designed a rectifier based on a single organic molecule [1], then the era of molecular devices was coming. Many important breakthroughs have been achieved in molecular devices already [2-8]. By means of external electric field, deformations of the molecular and light incident, many intriguing properties have been acquired in the past years [9-11], such as negative differential resistance (NDR) [12-21], molecular rectification [1,22-25], molecular switch [17,26-28], and

Guojun $\mathrm{Jin}^{\dagger}$, Hai Yang*

E-mail: gjin@nju.edu.cn, kmyangh@263.net

1 School of Physical Science and Technology, Kunming University, Kunming 650214, China

2 School of Physics and Astronomy, Yunnan University, Kunming 650504, China

3 National Laboratory of Solid State Microstructures, Department of Physics, and Collaborative Innovation Center of Advanced Microstructures, Nanjing University, Nanjing 210093, China 
conductance enhancement [29-30]. Some related works [31-34], early reported, are of great value to promote the realization of quantum computer.

In 2004, Novoselov and Geim et al. [35] prepared graphene by micromechanical separation, then graphene-based electrodes are widely used in many molecular devices, due to its excellent properties, such as long spin diffusion length, spin relaxation time, and high spin injection ratio [36-41]. Graphene nanoribbons (GNRs) are two-dimensional honeycomb structures composed of carbon atoms, and their widths are less than $10 \mathrm{~nm}$. They can be divided into zigzag graphene nanoribbons (ZGNR) and armchair graphene nanoribbons (AGNR) according to their different structures edge. GNRs, consisting of $s p^{2}$ hybridized carbon atoms, are touted as the fundamental basis for the next generation of electronic, spintronic and photonic devices.

Phenanthrene (PHE) is a thick aromatic hydrocarbon containing three benzene rings, with molecular formula $\mathrm{C}_{14} \mathrm{H}_{10}$, can potentially be used to model the interactions of aromatic systems. PHE has a very wide range of applications in the medical, environmental management and dye industries [42-47]. Due to the high specific surface area $\left(2600 \mathrm{~m}^{2} / \mathrm{g}\right)$ and good electrical conductivity $(7200 \mathrm{~S} / \mathrm{m})$ [48], a graphene sheet has a higher adsorption capacity than carbon nanotube and graphite for PHE. The molecular devices, in which the PHE chemically adsorbed to metal electrodes, have many interesting properties [45,49-50].

In this work, using density functional theory combined with non-equilibrium Green's function method, we have investigated the quantum transport properties of three different coupling configurations consisting of PHE and ZGNR. The dispersion relations, transmission spectrum, differential conductance $(\mathrm{d} I / \mathrm{d} V)$, current and voltage $(I-V)$ curves, and molecular projection self-consistent Hamiltonian (MPSH), which are closely related to quantum transport properties, are systematically calculated. Our results indicate that the proposed molecular device exhibits the outstanding NDR and odd-even effect. Thus, we assume that the novel PHE coupled ZGNR structure can be a promising molecular device in the future.

\section{Models and methods}

This work is based on the model fusing of a PHE to ZGNR, as shown in Fig. 1. The gray and white atoms are carbon and hydrogen, respectively. The six carbon atoms in the PHE molecule are labelled as L-Q in sequential order in Fig. 1(a), and the $\mathrm{N}-\mathrm{Q}$ and L-M positions are linked to the ZGNR. After dehydrogenation of carbon atoms in N-Q positions, the PHE is covalently coupled with the carbon atoms at the edge of ZGNR to form four C-C bonds (Fig. 1(b)). The hybrid structure is labelled as 3-ZGNR@PHE means the PHE links to $W$-ZGNR $(W=3)$, where the number $W$ (3) indicates the number of the zigzag carbon chains (ZCCs) along the $x$-axis. The other two coupling configurations are shown in Fig. 1(c) and Fig. $1(\mathrm{~d})$. Figure $1(\mathrm{~d})$ displays that the PHE rotates 90 degrees around the $x$-axis, then covalently couple with the two nearest carbon atoms in ZGNR, and the $s p^{3}$ hybrid emerges. For the sake of convenience, the configurations consisting of the PHE and ZGNR in Figs. 1(b)-1(d) are identified as A, B, and C, respectively, and $\mathrm{X}$ stands for $\mathrm{A}, \mathrm{B}$, and $\mathrm{C}$, thus the three hybrid configurations are named as 3-ZGNR@PHE(A), 3-ZGNR@PHE(B), and 3-ZGNR@PHE(C). Based on the above covalent link between the PHE and ZGNR, the corresponding specific two- 


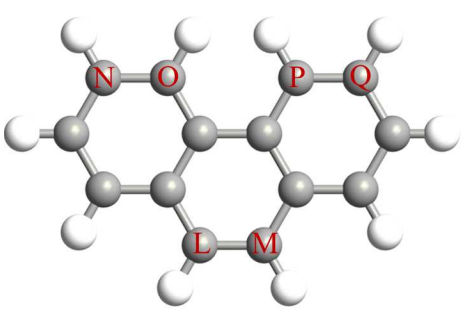

(a)

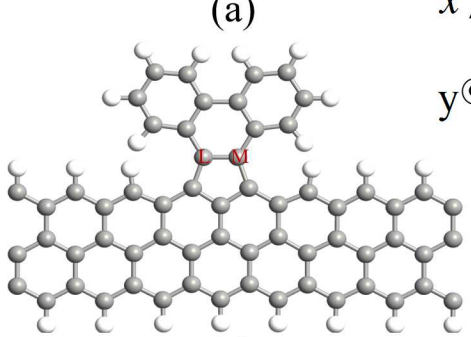

(c)

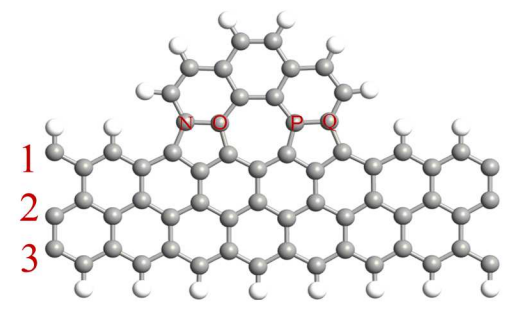

(b)<smiles>[3H][As]C</smiles>

\section{$\$$}

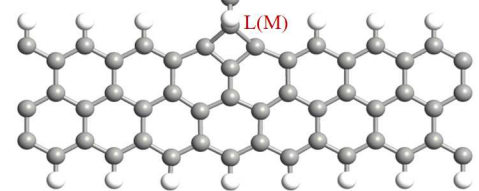

(d)

Fig. 1 The geometrical structures of the ZGNR decorated with a PHE molecule. (a) A PHE molecule $\left(\mathrm{C}_{14} \mathrm{H}_{10}\right)$. (b) The four C-C bonds at N, O, P, and Q positions connecting the PHE and ZGNR, named as configuration A. (c) The two C-C bonds at L and M positions, labelled as configuration B. (d) Configuration C, originating from (c) by rotation of 90 degree along the $x$-axis.

probe hybrid structures $(W=3,4,5,6)$ are constructed in the top three rows of Figs. 2(a)-(d). In order to stabilize the hybrid structure, hydrogen atom is used to eliminate the hanging bonds. In the hybrid structures of covalent coupling between the PHE and ZGNR, ZGNR is composed of 12 basic unit cells. Each of devices is divided into three parts: the left electrode (LE), the central scattering region (CSR), and the right electrode (RE). The LE and RE are denoted by the red and blue shadow, respectively, as shown in Fig. 2.

Quantum transport calculations for the three combinations $W$-ZGNG@PHE(X) are performed using the density functional theory (DFT) with the nonequilibrium Green's function (NEGF) implemented in the ATOMISTIX TOOLKIT (ATK) package [51-52]. The exchange-correlation function is described by Perdew-BurkeErnzerhof under generalized gradient approximation (GGA-PBE). A large enough vacuum $(>15 \AA)$ in the non-electron transmission direction $(x / y)$ is adopted to eliminate interactions between periodic images. All Carbon and hydrogen atoms are fully relaxed by using the quasi-Newton method until the energy reaches $10^{-5} \mathrm{eV}$ and the force on each atom is less than $0.05 \mathrm{eV} / \AA$. For the valence electrons of hybrid structures are adopted to double- $\zeta$ plus polarization (DZP) basis. The cut-off energy of real-space grid is $150 \mathrm{Ry}$. The Brillouin zone is sampled with a $1 \times 1 \times 100$ mesh of k-points.

The expression of the transmission coefficient at zero bias is

$$
T\left(E, V_{b}\right)=\operatorname{Tr}\left[\Gamma_{\mathrm{l}} G^{\mathrm{R}} \Gamma_{\mathrm{r}} G^{\mathrm{A}}\right],
$$

where $V_{b}$ is the bias voltage. $G^{\mathrm{R}}$ and $G^{\mathrm{A}}$ are the retarded and advanced Green's functions of the CSR, respectively. $\Gamma_{1}$ and $\Gamma_{\mathrm{r}}$ are the broadening matrix of LE and RE, respectively. Under finite bias, $I-V$ curves of the hybrid structures can 


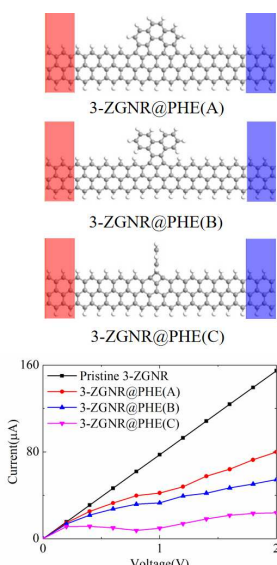

(a)

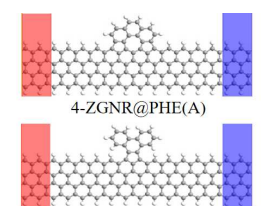

4-ZGNR@PHE(B)

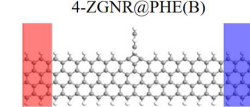

4-ZGNR@PHE(C)

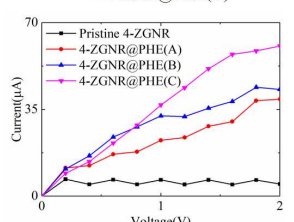

(b)

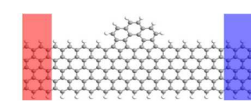

5-ZGNR@PHE(A)

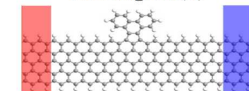

5-ZGNR@PHE(B)

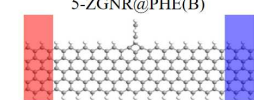

5-ZGNR@PHE(C)

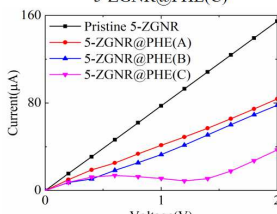

(c)

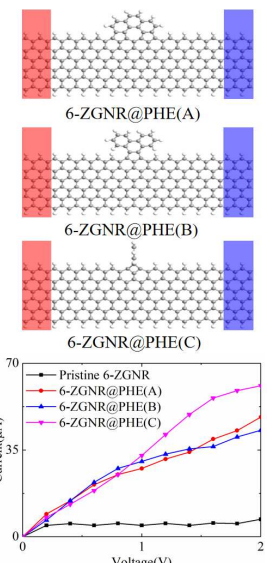

(d)

Fig. 2 Calculated currents as a function of the applied bias and the number of carbon chains across the ribbon width for ZGNRs-based hybrid structures and pristine ZGNR. The first three rows in each graph show the geometrical structures of the two-probe systems for the three different PHE/ZGNR coupling configurations, which are labelled as $W$-ZGNR@PHE(X) ( $W=3,5,4$, and $6, \mathrm{X}=\mathrm{A}, \mathrm{B}$, and $\mathrm{C})$. (a) The $I-V$ characteristics of 3 -ZGNR@PHE(X). (b) The $I-V$ characteristics of 4 -ZGNR@PHE(X). (c) The $I-V$ characteristics of 5-ZGNR@PHE(X). (d) The $I-V$ characteristics of 6-ZGNR@PHE(X).

be calculated using the Lander-Büttiker formula in mesoscopic physics [53] as

$$
I\left(V_{b}\right)=\frac{2 e}{h} \int T\left(E, V_{b}\right)\left[f_{1}\left(E-\mu_{1}\right)-f_{\mathrm{r}}\left(E-\mu_{\mathrm{r}}\right)\right] d E,
$$

where $\mu_{\mathrm{l}(\mathrm{r})}=E_{\mathrm{F}} \pm e V_{b} / 2$ is the chemical potential of the LE and RE, $E_{\mathrm{F}}$ is the Fermi level, which is set to zero here. $f_{1(\mathrm{r})}$ is the Fermi distribution function of the left (right) electrode.

\section{Results and discussion}

We start with currents as a function of applied bias for pristine $W$-ZGNR and their hybrid structures (the number of carbon chains cross the ribbon width of ZGNR, $W=3,4,5,6$ ) (Fig. 2). Firstly, the $I$ - $V$ curve of pristine 3-ZGNR shows excellent linearity (Fig. 2(a)), by contrast, the three PHE coupled 3-ZGNR hybrid structures all exhibit the current reduction under the whole positive bias. It has been obtained that the $I$ - $V$ curves of ZGNR with the odd number of ZCCs have linear growth because of wavefunctions without the mirror symmetry about the yz middle plane, which is also well verified here $[18,54]$. For 3-ZGNR@PHE(C), with the voltage increases, the current initially increases until reaches the peak value at $0.4 \mathrm{~V}$. As the voltage continues to increase, its current decreases. This reveals that $I$ - $V$ of 3 -ZGNR@PHE(C) has NDR at low bias voltage. NDR has a wide range of applications in molecular electronics in the future, such as oscillators, memories, amplifiers and logic circuits. Secondly, compared with 3-ZGNR@PHE(X) hybrid structures, the results of 4-ZGNR@PHE(X) are totally different. It is observed that the current of the pristine 4 -ZGNR varies little with the change of 


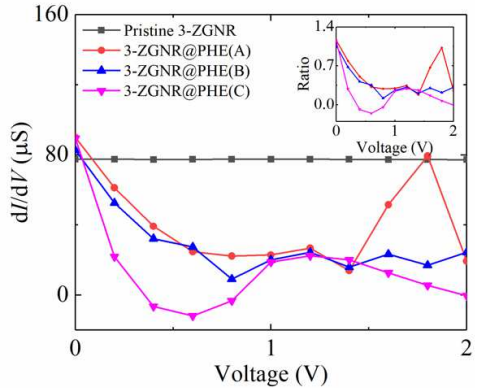

(a)

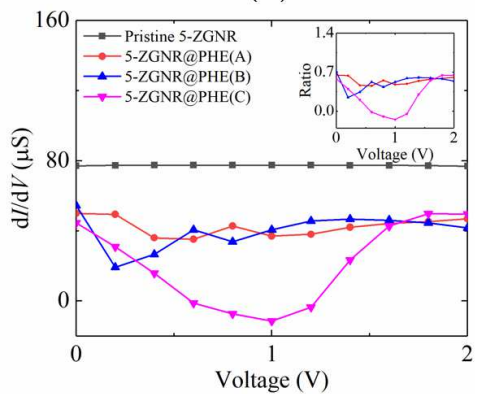

(c)

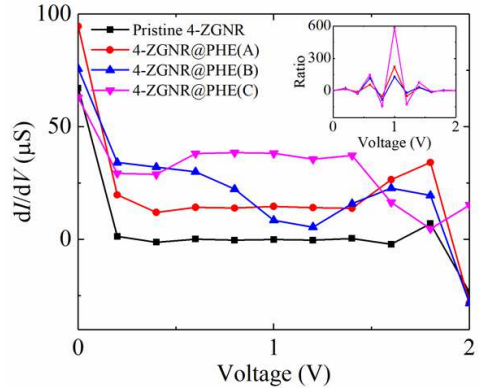

(b)

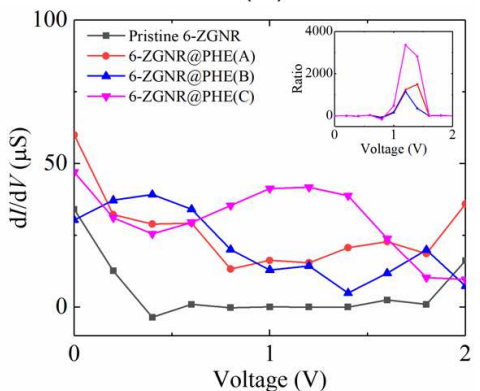

(d)

Fig. 3 Calculated $\mathrm{d} I / \mathrm{d} V$ s of the pristine ZGNR and the hybrid configurations. (a), (b), (c), and (d) The $\mathrm{d} I / \mathrm{d} V \mathrm{~s}$ of the ZGNR and 3-ZGNR@PHE(X), 4-ZGNR@PHE(X), 5ZGNR@PHE(X), and 6-ZGNR@PHE(X), respectively. The $\mathrm{d} I / \mathrm{d} V$ ratios are shown in the insets.

voltage, and stabilizes at a fixed value (about $6 \mu \mathrm{A}$ ). However, for PHE fused 4-ZGNR hybrid structures, the currents increase with the increase of bias voltage. Especially, the current values of 4-ZGNR@PHE(C) are almost linear with the increase of bias voltage in this region $[0.2,1.6 \mathrm{~V}]$. Thirdly, the behaviors of the 5-ZGNR related hybrid structures reveal a striking resemblance to those of 3ZGNR@PHE(X) (Figs. 2(a) and 2(c)). The currents of 5-ZGNR@PHE(X) is less than that of the pristine 5-ZGNR. Finally, Fig. 2(d) displays the calculated $I-V$ curves of pristine 6-ZGNR and its related hybrid structures. The currents of the 6ZGNR@PHE(X) is significantly enhanced due to electrons enter the left electrode through the barrier tunneling. Consequently, we conclude that the results of odd (even)-ZGNR@PHE(X) are very similar.

Next, we observe the rule of $\mathrm{d} I / \mathrm{d} V$ of all hybrid structures via the $\mathrm{d} I / \mathrm{d} V$ ratio, which is plotted in Fig. 3. The $\mathrm{d} I / \mathrm{d} V$ is defined as

$$
G=d I / d V
$$

$I$ and $V$ represent the current and voltage values, respectively, thereby the $\mathrm{d} I / \mathrm{d} V$ ratio is defined as (illustrated in Fig. 3)

$$
\text { Ratio }=G / G_{\text {Pristine }}
$$

where $G_{\text {Pristine }}$ and $G$ represent the $\mathrm{d} I / \mathrm{d} V$ s values of pristine $W$-ZGNR and $W$-ZGNR@PHE(X), respectively. The $\mathrm{d} I / \mathrm{d} V$ s of pristine odd-ZGNR (odd=3, 5 ) 


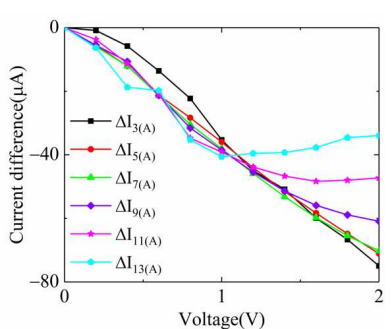

(a)

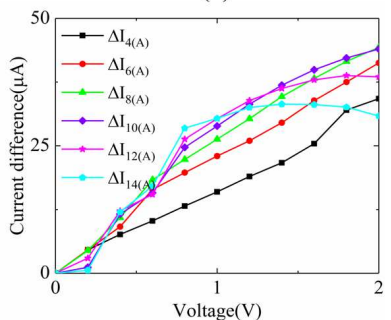

(d)

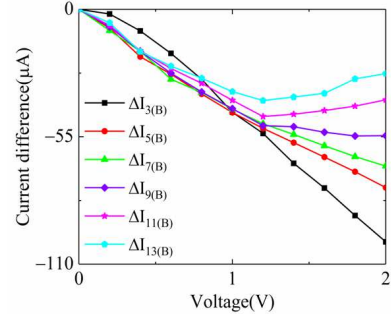

(b)

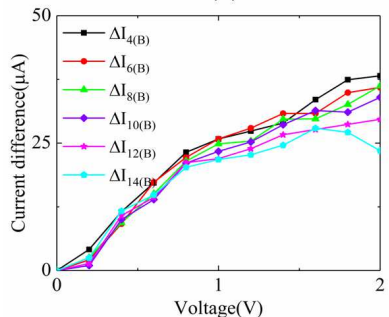

(e)

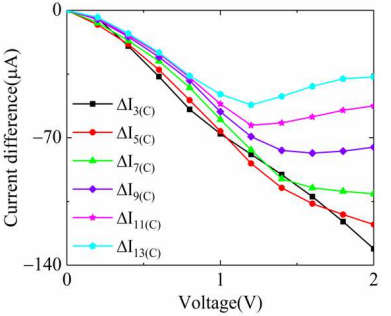

(c)

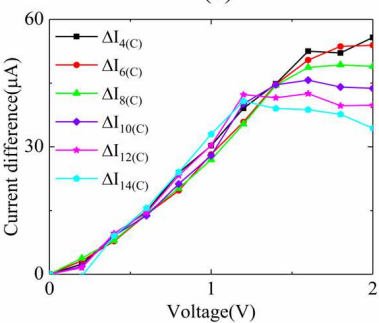

(f)

Fig. 4 The effect of the odd (even) number of ZCCs across the ribbon width and the applied bias on the $\triangle I$ for ZGNRs-based hybrid structures and pristine graphene. (a)-(c) The effect of the odd number of ZCCs and the applied bias on the $\triangle I$ for ZGNRs-based hybrid structures and pristine graphene. (b)-(f) The effect of the the even number of ZCCs and the applied bias on the $\triangle I$ for ZGNRs-based hybrid structures and pristine graphene.

are constant (about $78 \mu \mathrm{S}$ ), as shown in Figs. 3(a) and 3(c). Nevertheless, the $\mathrm{d} I / \mathrm{d} V \mathrm{~s}$ of odd-ZGNR@PHE(X) are significantly less than ones of pristine oddZGNR. Besides, the $\mathrm{d} I / \mathrm{d} V$ ratios for odd-ZGNR@PHE(X) are less than 1, which indicate that $\mathrm{d} I / \mathrm{d} V$ s can be weakened greatly in the fusing of PHE to odd-ZGNR. Moreover, the $\mathrm{d} I / \mathrm{d} V \mathrm{~s}$ of $3(5)-Z G N R @ P H E(C)$ is less than 0 at $[0.4,0.8 \mathrm{~V}]([0.6$, $1.2 \mathrm{~V}]$ ), which means that the slope of $I-V$ is negative, thus the NDR is verified again. The $\mathrm{d} I / \mathrm{d} V \mathrm{~s}$ of even-ZGNR is almost zero at $[0.4,1.8 \mathrm{~V}]$ (Figs. 3(b) and $3(\mathrm{~d}))$. However, the $\mathrm{d} I / \mathrm{d} V$ s of even-ZGNR@PHE(X) are all above it. Especially, 4 (6)-ZGNR@PHE(C) exhibits excellent conductance enhancement characteristics, which leads the $\mathrm{d} I / \mathrm{d} V \mathrm{~s}$ of 4 (6)-ZGNR@PHE(C) to be 581 (3480) times of the pristine 4 (6)-ZGNR at $1.0(1.2) \mathrm{V}$.

Clearly, the number of carbon dimer lines across the ribbon width determines the conductances of this molecule device. By decorating with the PHE, the conductance decreases when the number of ZCCs of ZGNR is odd, however, the conductance increases when the number of ZCCs is even, which revealing odd-even effect. In order to further verify the parity of the hybrid structures, we have also calculated the $I-V$ curves of other eight hybrid structures (e.g., $W$-ZGNR@PHE(X) $(W=7,8,9,10,11,12,13,14))$. Here we adopted the current difference $(\triangle I)$, for observing the effect of parity with the increase of the number ZCCs (Figs. 4(a)-(f)). The $\triangle I$ is defined as

$$
\triangle I_{W(\mathrm{X})}=I_{W-\mathrm{ZGNR} @ \mathrm{PHE}(\mathrm{X})}-I_{\text {Pristine } W-\mathrm{ZGNR}},
$$

based on equation (5), when $\triangle I$ is positive, the currents of $W$-ZGNR@PHR(X) increases, otherwise it decreases. In Figs. 4(a)-(c), $\triangle I_{\text {odd (X) }}$ are all negative, which 

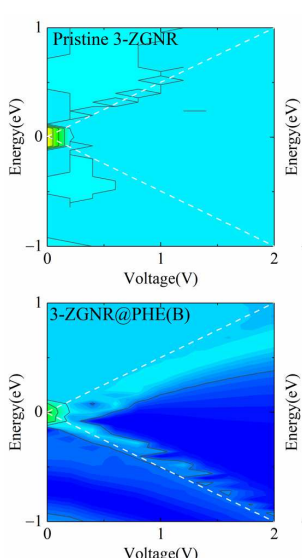

Voltage(V)

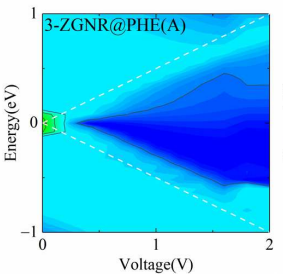

Voltage(V)

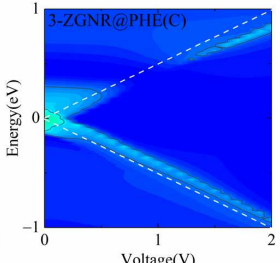

(a)
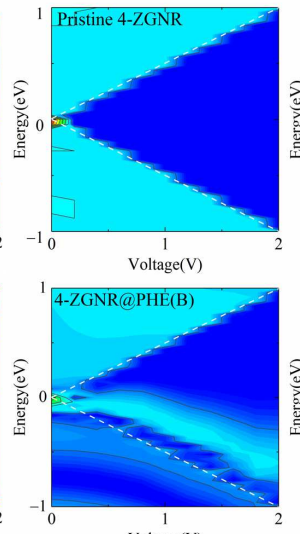
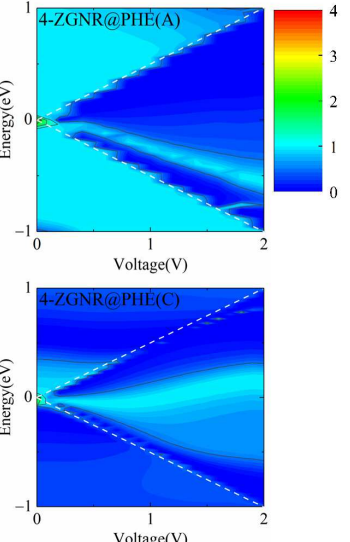

(b)

Fig. 5 Transmission spectra as a function of electron energy and bias voltages for (a) 3ZGNR@PHE(X) and (b) 4-ZGNR@PHE(X). The region between the two white dotted lines is the bias window.

shows the decrease of conductance for the PHE fused the odd number of ZCCs hybrid structures. In the voltage range of 0-1.2 V, the $\left|\triangle I_{\text {odd }(\mathrm{X})}\right|$ increases, however, when the voltage is beyond $1.2 \mathrm{~V}$, especially at $2 \mathrm{~V},\left|\triangle I_{\mathrm{odd}(\mathrm{X})}\right|$ decreases with increasing ZCCs. For the PHE fused even-ZGNR, as shown in Figs. 4(d)-(f), $\left|\triangle I_{\text {even(X) }}\right|$ are positive which leads to an enhancement of the $\mathrm{d} I / \mathrm{d} V \mathrm{~s}$. And with the increase of ZCCs, $\left|\triangle I_{\text {even(X) }}\right|$ presents a decreasing trend. Besides, $\left|\triangle I_{W(X)}\right|$ reveals a decreasing trend after $1.2 \mathrm{~V}$, which indicates that when ZCCs increase to a certain value, the odd-even disappeared.

Thirdly, considering the transmission spectra as a function of electron energy and bias voltages as examples, we explained the origin of the current values of odd (even)-ZGNR@PHE(X) decrease (increase) relative to the pristine odd (even)ZGNR. We take the Fermi level as the energy reference point and the size of the bias window ranges from $-\frac{V_{b}}{2}$ to $+\frac{V_{b}}{2} \mathrm{eV}$ (white dotted line, as shown in Fig. 5). Based on Landauer-Büttiker formula, we found that the current of the devices can be obtained by integrating the transmission coefficient in the bias window. In Fig. 5(a), the transmission of pristine 3-ZGNR is almost 1 under the whole bias window. As the bias voltage increases, the bias window becomes wider, and the integral coefficient in the window increases. Thus, the current values of the pristine 3-ZGNR present good linearity. The transmission peaks of 3-ZGNR@PHE(X) only appear near the bias window, revealing that the conductance of 3-ZGNR@PHE(X) decreases. When the bias voltages change within the range of $[0.6,1.4 \mathrm{~V}]$, the peak value of the positive bias window of 3-ZGNR@PHE $(\mathrm{C})$ disappears gradually, leading to the decrease of current and the appearance of the NDR effect.

The reason for the conductance enhancement can be understood in terms of the transmission spectra of 4-ZGNR related device systems which are plotted in Fig. 5(b). The transmission of the pristine $4-Z$ GNR is 0 in the range of $\left[-\frac{V_{b}}{2}+\right.$ $\left.0.1,+\frac{V_{b}}{2}-0.1 \mathrm{eV}\right]$, but it is very close 1 within the scope of $\left(\left[-\frac{V_{b}}{2},-\frac{V_{b}}{2}+0.1 \mathrm{eV}\right]\right.$ and $\left.\left[+\frac{V_{b}}{2}-0.1,+\frac{V_{b}}{2} \mathrm{eV}\right]\right)$, which ensure that the current values of pristine 4-ZGNR are about stable at $6 \mu \mathrm{A}$. For the pristine 4 -ZGNR, the electron wavefunctions of 


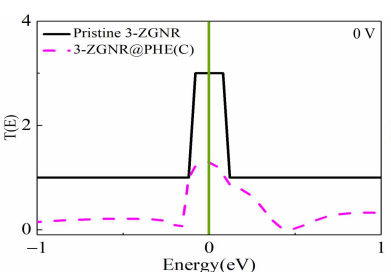

(a)

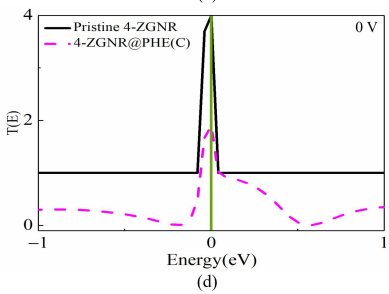

(d)

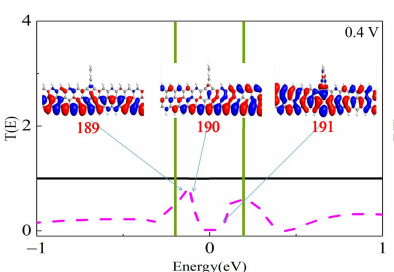

(b)

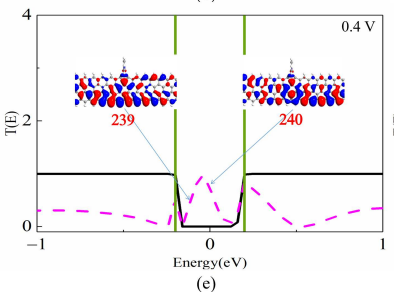

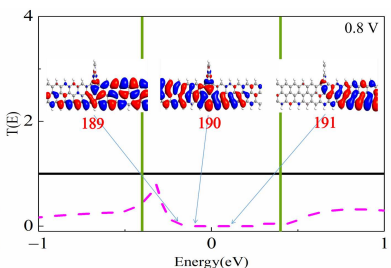

(c)

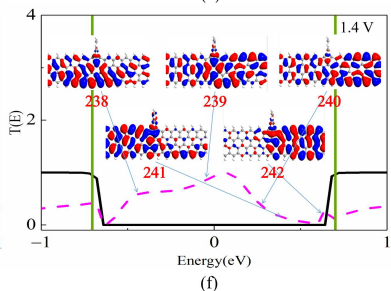

Fig. 6 Transmission spectra of pristine 3 (4)-ZGNR and 3 (4)-ZGNR@PHE(C) as a function of energy and bias voltages. (a) Bias voltage $0 \mathrm{~V}$, (b) $0.4 \mathrm{~V}$, and (c) $0.8 \mathrm{~V}$, (a)-(c) for 3-ZGNR and 3-ZGNR@PHE(C), the illustrations of (b) and (c) are the MPSHs in the bias windows. (d) Bias voltage $0 \mathrm{~V}$, (e) $0.4 \mathrm{~V}$, and (f) $1.4 \mathrm{~V}$, (d)-(f) for 4-ZGNR and 4-ZGNR@PHE(C), and the illustrations of (e) and (f) are the MPSHs in the bias windows, the green solid line is the bias window.

LE matches RE at the bias voltage of $0 \mathrm{~V}$. When the bias voltage increases, the energy band of LE and RE move to the low energy and high energy region, respectively, which makes the $\pi^{*}(\pi)$ band of LE and the $\pi\left(\pi^{*}\right)$ band of RE mismatch in the rang of $\left[-\frac{V_{b}}{2}+0.1,+\frac{V_{b}}{2}-0.1 \mathrm{eV}\right]$ (Fig. S1, as shown in the supporting information). When the PHE is anchored at the edges of 4-ZGNR, the pristine mirror symmetry is destroyed, symmetry laws are inapplicable any more. Within the range of the middle parts of the bias window, the transmission of the PHE fused 4-ZGNR increases obviously and close to 1 . Therefore, the fact that the transmission strengths of 4-ZGNR@PHE(X) are greater than that of pristine 4-ZGNR causes the currents growth. Similarly, the other $I$ - $V$ curves in Fig. 2 can be interpreted by using these transmission spectra, respectively.

Then we consider the NDR and odd-even. Figs. 6(a)-(c) reveal the transmission spectra of the pristine 3-ZGNR and 3-ZGNR@PHE(C) measured by twoprobe devices at $0 \mathrm{~V}, 0.4 \mathrm{~V}$, and $0.8 \mathrm{~V}$. The molecular projected self-consistent Hamiltonians (MPSHs) of 3-ZGNR@PHE(C) at $0.4 \mathrm{~V}$ and $0.8 \mathrm{~V}$ are also inserted in (b) and (c) respectively. At $0 \mathrm{~V}$ (Figs. 6(a) and 6(d)), the transmission peaks near the Fermi surface all exceed 1, which indicates the device has good metal characteristics. Two transmission peaks can be observed in Figs. 6(b) and 6(c) where they are labelled as $P 1$ and $P 2$. When the bias voltage at $0.4 \mathrm{~V}, P 1$ and $P 2$ appear at the energy of $-0.12 \mathrm{eV}$ and $0.19 \mathrm{eV}$, respectively. As the bias voltage increases to $0.8 \mathrm{~V}, P 1$ and $P 2$ move to the low-energy region and the high-energy region respectively with the bias window expands. Meanwhile, the peak value of $P 2$ gradually decreases during this process. When $0.4 \mathrm{~V}$ bias voltage is applied, there are three transmission channels in the bias window: 189, 190, 191 (Fig. 6(b) and $6(\mathrm{c}))$. The three orbits of 189-191 are delocalized over the whole device, thus the transmission channel is opened. The delocalization of the 189 and 190 transmission channels ensure that $P 1$ remains a sharp peak below the Fermi surface. 


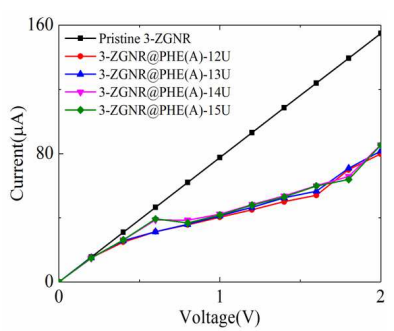

(a)

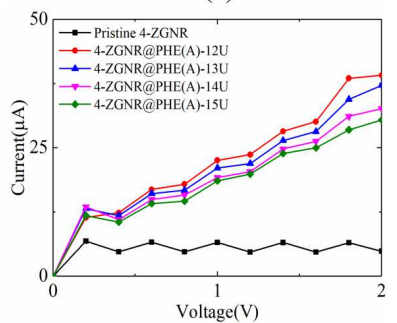

(d)

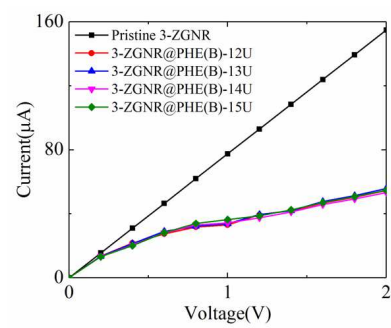

(b)

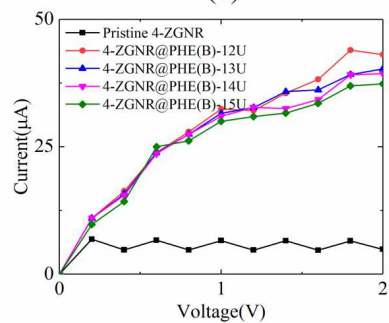

(e)

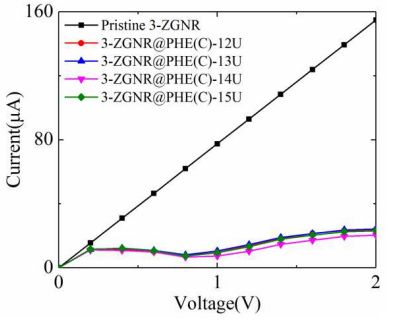

(c)

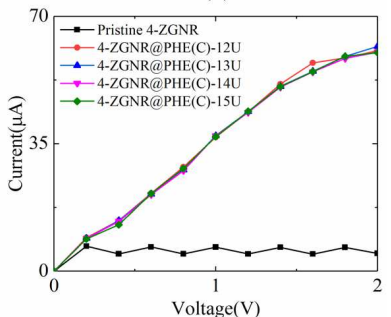

(f)

Fig. 7 (a)-(c) The $I-V$ of 3-ZGNR@PHE(X) for the different lengths of CSR (12 U, $13 \mathrm{U}$, $14 \mathrm{U}, 15 \mathrm{U})$. (d)-(f) The $I-V$ of 4 -ZGNR@PHE(X) for the different lengths of CSR.

With the applied bias voltage changes from $0.4 \mathrm{~V}$ to $0.8 \mathrm{~V}$, the localization of the 191 orbit is on the right electrode induces a gradual decrease of their corresponding transmission peak $P 2$. This is the cause of the NDR effect. In addition, with the bias voltage increasing, the number of orbits remains the same and the delocalization of the 189-191 orbits on the device is insignificant, which makes the transmission of 3-ZGNR@PHE(C) less than that of pristine 3-ZGNR, thereby causing the decrease of conductance.

For the case of PHE fused even-ZGNR, we adopt 4-ZGNR@PHE(C) as a representative to explore the conductance enhancement. Figs. 6(d)-(f) show the calculated transmission spectra of the pristine 4-ZGNR and 4-ZGNR@PHE(C) at $0 \mathrm{~V}, 0.4 \mathrm{~V}$ and $1.4 \mathrm{~V}$. The MPSHs of 4-ZGNR@PHE $(\mathrm{C})$ are also added in Figs. $6(\mathrm{e})$ and $6(\mathrm{f})$, respectively. For pristine $4-\mathrm{ZGNR}$, in the range of $0.1 \mathrm{eV}$ at the bias window, a transmission peak of 1 appears, which makes the current fixed at $6 \mu \mathrm{A}$. However, 4-ZGNR@PHE(C) exhibits a higher and wider transmission peak due to the broken symmetry of structure in the bias window. Compared with the transmission coefficient under the bias voltage of $0.4 \mathrm{~V}$, the transmission peak at $1.4 \mathrm{~V}$ becomes wider and flatter, which leads to the integrated area of the transmission coefficient at $1.4 \mathrm{~V}$ within the bias window is much larger than that under $0.4 \mathrm{~V}$. When the bias of $0.4 \mathrm{~V}$ is applied, only the molecular orbits 239 and 240 reveal delocalization. The delocalized states of the orbits 239 and 240 cause a sharp transmission peak at the Fermi level. However, there are other transmission channels at $1.4 \mathrm{~V}$ (e.g., 238, 239, 240, 241, and 242). More transmission channels make the transmission peak larger and wider at $1.4 \mathrm{~V}$.

Finally, to make the research process more systematic, it is essential to study the effect of the CSR lengths on electron transport of 3 (4)-ZGNR and 3 (4)ZGNR@PHE(X). Fig. 7 displays the calculated $I-V$ curves for the various hybrid structures with different lengths (where ZGNR is 12, 13, 14, and 15 unit cells). We 
find that, with altering the lengths of CSR, the $I-V$ curves of 3-ZGNR@PHE(X) revealing a similar variation tendency, and the current is basically equal. Furthermore, when the CSR gradually increases, the current of 4-ZGNR@PHE(B) and 4-ZGNR@PHE(C) is basically unchanged. Accordingly, we suggest that, regardless of the change in the lengths of the CSR, it will hardly affect the current and parity characteristics of 3-ZGNR@PHE(X) and 4-ZGNR@PHE(X).

Overall, our study indicates that the quantum transport properties of these hybrid structure are mainly determined by the number of ZCCs across the ribbon width, rather than the ribbon length.

\section{Summary}

In this paper, we systematically studied the nanoscopic transport properties of the fusing of the PHE to ZGNR by using a first-principle calculation based on DFT and NEGF. The results show that, firstly, $W$-ZGNR@PHE(C) $(W=3,5,7,9)$ has an NDR effect. On a deep physical level, when the bias is at $[0.4,0.8 \mathrm{~V}]$, the electrons in the orbital 191 are gradually localized to the right electrode, which makes the gradual disappearance of the transmission peak $P 2$, thereby supplying the production of the NDR effect. Secondly, the width of the carbon dimer line determines the conductance of the molecule device. Specifically, when PHE coupling to odd (even)-ZGNR, the conductance of the molecule device decreases (increases) and shows odd-even characteristics. The reason for the parity is that compared with the pristine odd (even)-ZGNR, after coupling PHE, the transmissions of the bias window decrease (increase). In brief, our results illuminate that the PHE coupled ZGNR hybrid structures have a high application value in the field of molecular electronics (e.g., oscillators, amplifiers, memories, and logic circuits et al.).

Acknowledgements This work was supported by the National Nature Science Foundation of China (Grant Nos. 11264022, 11874168, and 12074156), the Yunnan Local College Applied Basic Research Projects (Grants Nos. 2017FH001-001, 2018FH001-016, and 2019FH001-077), the General Projects of Yunnan Education Department(No. 2018JS393). We also acknowledge the support of the National Laboratory of Solid State Microstructures (No. M31020 and M32063), Nanjing University, Nanjing 210093, China.

\section{References}

1. Aviram, A., Ratner, M. A.: Molecular Rectifiers. Chem. Phys. Lett. 29, 277-283 (1974)

2. Stokbro, K., Taylor, J., Brandbyge, M., Mozos, J.-L., Ordejón, P.: Theoretical study of the nonlinear conductance of Di-thiol benzene coupled to $\mathrm{Au}(111)$ surfaces via thiol and thiolate bonds. Comp. Mater. Sci. 27, 151-160 (2003)

3. Xu, K., Huang, J., Guan, Z., Li, Q., Yang, J.: Transport spin polarization of magnetic $C_{28}$ molecular junctions. Chem. Phys. Lett. 535, 111-115 (2012)

4. Han, J., Feng, Y., Yao, K., Gao, G.Y.: Spin transport properties based on spin gapless semiconductor CoFeMnSi. Appl. Phys. Lett. 111, 132402 (2017)

5. Markevich, A., Kurasch, S., Lehtinen, O., Reimer, O., Feng, X., Müllen, K., Turchanin, A., Khlobystov, A.N, Kaiser, U., Besley, E.: Electron beam controlled covalent attachment of small organic molecules to graphene. Nanoscale 8, 2711-2719 (2016)

6. Su, T.A., Neupane, M., Steigerwald, M.L., Venkataraman, L., Nuckolls, C.:Chemical principles of single-molecule electronics. Nat. Rev. Mater. 1, 1-15 (2016) 
7. Smith, S., Llinás, J.P., Bokor, J., Salahuddin, S.:Negative Differential Resistance and Steep Switching in Chevron Graphene Nanoribbon Field-Effect Transistors. Ieee. Electr. Device L. 39, 143-146 (2018)

8. Nakabayashi, J., Yamamoto, D., Kurihara, S.:Band-Selective Filter in a Zigzag Graphene Nanoribbon. Phys. Rev. Lett. 102, 066803 (2009)

9. Žutić, I., Fabian, J., Sarma, S.D.: Spintronics: Fundamentals and applications. Rev. Mod. Phys. 76, 323-410 (2004)

10. Mathew, P.T., Fang, F.: Advances in Molecular Electronics: A Brief Review. EngineeringPRC 4, 760-771 (2018)

11. Fan, Z.Q., Sun, W.Y., Jiang, X.W., Zhang, Z.H., Deng, X.Q., Tang, G.P., Xie, H.Q., Long, M.Q.: Redox control of magnetic transport properties of a single anthraquinone molecule with different contacted geometries. Carbon, 113, 18-25 (2017).

12. Long, M.Q., Chen, K.Q., Wang, L., Zou, B.S., Shuai, Z.: Negative differential resistance induced by intermolecular interaction in a bimolecular device. Appl. Phys. Lett. 91, 233512 (2007)

13. Zhao, P., Gao, X.J., Song, Y., Li, X.X., Chen, G.: Spin-filtering, giant magnetoresistance, negative differential resistance effects and spin logic gate in $P_{2} T A-O_{2}-$ based molecular junction with different transition metal atoms. Org. Electron. 57, 104-109 (2018)

14. Müller, M., Luysberg, M., Schneider, C.M.: Observation of spin filtering in magnetic insulator contacts to silicon. Appl. Phys. Lett. 98, 142503 (2011)

15. Zeng, M.G., Shen, L., Cai, Y.Q., Sha, Z.D., Feng, Y.P.: Perfect spin-filter and spin-valve in carbon atomic chains. Appl. Phys. Lett. 96, 042104 (2010)

16. Ni, Y., Yao, K.L., Tang, C.Q., Gao, G.Y., Fu, H.H., Zhu, S.C.: Perfect spin-filter, spinvalve, switching and negative differential resistance in an organic molecular device with graphene leads. Rsc Adv. 4, 18522 (2014)

17. Kumar, M.: Negative differential resistance and switching behavior in single wall bambooshape carbon nanotubes based molecular device: A first-principles study nanoscale device design. Mater. Res. Bull. 91, 148-154 (2017)

18. Zeng, J., Chen, K.Q., Tong, Y.X.: Covalent coupling of porphines to graphene edges: Quantum transport properties and their applications in electronics. Carbon 127, 611-617 (2018)

19. Li, S., Liu, X., Nandi, S.K., Nath, S.K., Elliman, R.G.:Origin of current-controlled negative differential resistance modes and the emergence of composite characteristics with high complexity. Adv. Funct. Mater. 29, 1905060 (2019)

20. Wu, D., Cao, X.H., Chen, S.Z., Tang, L.M., Feng, Y.X., Chen,K.Q., Zhou, W.X.: Pure spin current generated in thermally driven molecular magnetic junctions: a promising mechanism for thermoelectric conversion. J. Mater. Chem. A, 7, 19037 (2019)

21. Zhang, Z., Guo, C., Kwong, D.J., Li, J., Deng, X., Fan, Z.: A dramatic odd-even oscillating behavior for the current rectification and negative differential resistance in carbon-chainmodified donor-acceptor molecular devices. Adv. Funct. Mater. 23, 2765-2774 (2013)

22. Wang, Z.Q., Wei, M.Z., Dong, M.M., Hu, G.C., Li, Z.L., Wang, C.K., Zhang, G.P.: HighPerformance Single-Molecule Switch Designed by Changing Parity of Electronic Wave Functions via Intramolecular Proton Transfer. J. Phys. Chem.C 122, 17650-17695 (2018)

23. Song, Y., Xie, Z., Ma, Y., Li, Z.L., Wang, C.K.: Giant Rectification Ratios of Azulenelike Dipole Molecular Junctions Induced by Chemical Doping in Armchair-Edged Graphene Nanoribbon Electrodes. J. Phys. Chem.C 118, 18713-18720 (2014)

24. Wu, Q.H., Zhao, P., Liu,H.Y., Liu, D.S., Chen, G.: OddCeven dependence of rectifying behavior in carbon chains modified diphenyl-dimethyl molecule. Chem. Phys. Lett. 605, 62-66 (2014)

25. Vezzoli, A., Brooke, R.J., Higgins, S.J., Schwarzacher, W., Nichols, R.J.: Single-Molecule Photocurrent at a Metal-Molecule-Semiconductor Junction. Nano Lett. 17, 6702-6707 (2017)

26. Zhang, D., Long, M., Zhang, X., Xu, H.: High performance bipolar spin filtering and switching functions of poly-(terphenylene-butadiynylene) between zigzag graphene nanoribbon electrodes. Rsc Adv. 5, 96455 C96463 (2015)

27. Wan, H., Zhou, B., Chen, X., Sun, C.Q., Zhou, G.: Switching, Dual Spin-Filtering Effects, and Negative Differential Resistance in a Carbon-Based Molecular Device. J. Phys. Chem. C 116, 2570C2574 (2012)

28. Yan, Q.Y., Huang, B., Yu, J., Zheng, F., Zang, J., Wu, J., Gu, B.L., Liu, F., Duan, W.: Intrinsic current-voltage characteristics of graphene nanoribbon transistors and effect of edge doping. Nano Lett. 7, 1469-1473 (2007) 
29. Leary, E., Limbury, B., Alanazy, A., Sangtarash, S., Grace, I., Swada, K., Esdaile, L.J., Noori, M., González, M.T., Gabino, Rubio-Bollinger, G., Sadeghi, H., Hodgson, A., Agraït, N., Higgins, S.J., Lambert, C.J., Anderson, H.L., Nichols, R.J.: Bias-Driven Conductance Increase with Length in Porphyrin Tapes. J. Am. Chem. Soc. 140, 12877-12883 (2018)

30. Zhen, S., Mao, J.C., Chen, L., Ding, S., Luo, W., Zhou, X. S., Qin, A., Zhao, Z., Tang, B.Z.: Remarkable multichannel conductance of novel single-molecule wires built on through-space conjugated hexaphenylbenzene. Nano Lett. 7, 4200-4205 (2018)

31. Jiang, Z.F., Li, R.D., Zhang, S.C., Liu, W.M.: Semiclassical time evolution of the holes from Luttinger Hamiltonian. Phys. Rev. B 72, 045201 (2005)

32. Ji, A.C., Xie, X.C., Liu, W.M.: Quantum Magnetic Dynamics of Polarized Light in Arrays of Microcavities. Phys. Rev. Lett. 99, 183602 (2007)

33. Chen, Y.H., Tao, H.S., Yao, D.X., Liu, W.M.: Kondo Metal and Ferrimagnetic Insulator on the Triangular Kagome Lattice. Phys. Rev. Lett. 108, 246402 (2012)

34. Zhang, X.L., Liu, L.F., Liu, W.M.: Quantum Anomalous Hall Effect and Tunable Topological States in 3d Transition Metals Doped Silicene. Sci. Rep. 3, 2908 (2013)

35. Novoselov, K.S., Geim, A.K., Morozov, S. V., Jiang, D., Zhang, Y., Dubonos, S.V., Grigorieva, I.V., Firsov, A.A.: Electric field effect in atomically thin carbon films. Science 306, 666-669 (2004)

36. Geim, A.K., Novoselov, K.S.: The rise of graphene. Nat. Mater. 6, 183-191 (2007)

37. Novoselov, K.S.: Nobel Lecture: Graphene: Materials in the Flatland. Rev. Mod. Phys. 83, 837-849 (2011)

38. de Bruijckere, J., Gehring, P., Palacios-Corella, M., Clemente-León, M., Coronado, E., Paaske, J., Hedegård P., van der Zant, H.S.J: Ground-state spin blockade in a single-molecule junction. Phys. Rev. Lett. 122, 197701 (2019)

39. Li, X., Yang, J:First principles design of spintronics materials. Natl. Sci. Rev. 3, 365-381 (2016)

40. Liu, J., Berger, R., Müllen, K., Feng, X.: Nanographenes and Graphene Nanoribbons with Zigzag-Edged Structures. Adv. Polym. Sci. 278, 1-32 (2017)

41. Xin, N., Li, X., Jia, C., Gong, Y., Li, M., Wang, S., Zhang, G., Yang, J., Guo, X.: Tuning Charge Transport in AromaticCRing SingleCMolecule Junctions via Ionic Liquid Gating. Angew. Chem. Int. Edit. 57, 14026-14031 (2018)

42. Yang, K., Chen, B., Zhu, L.: Graphene-coated materials using silica particles as a framework for highly efficient removal of aromatic pollutants in water. SCI REP-UK. 5, 11641 (2015)

43. Zhang, Y., Liu, P., Li, Y., Zhan, R., Huang, Z., Lin, H.: Study on fluorescence spectroscopy of PAHs with different molecular structures using laser-induced fluorescence (LIF) measurement and TD-DFT calculation. Spectrochim. Acta. A. 224, 117450 (2019)

44. Alrikabi, A.: Theoretical study of the design dye-sensitivity for usage in the solar cell device. Results Phys. 7, 4359-4363 (2017)

45. Gao, Z., Liu, D., Tománek, D.: Two-Dimensional Mechanical Metamaterials with Unusual Poisson Ratio Behavior. Phys. Rev. Appl. 10, 064039 (2018)

46. Zhao,J., Wang, Z., Zhao, Q., Xing, B.: Adsorption of Phenanthrene on Multilayer Graphene as Affected by Surfactant and Exfoliation. Environ. Sci. Technol. 48, 331-339 (2014)

47. Cui, S., Huang, Q., Wang, J., Jia, H., Huang, P., Wang, S., Du, P.: From planar macrocycle to cylindrical molecule: synthesis and properties of a phenanthrene-based coronal nanohoop as a segment of $[6,6]$ carbon nanotube. Org. Lett. 21, 5917-5921 (2019)

48. Park, S., Ruoff, R.S.: Chemical methods for the production of graphenes. Nat. Nanotechol. 4, $217 \mathrm{C} 224(2009)$

49. Han, X., Yang, J., Yuan, P., Bian, B., Shi, H., Ding, Y.: Spin-dependent transport in a multifunctional spintronic device with graphene nanoribbon electrodes. J. Comput. Electron. 17, 604-612 (2018)

50. Razeghi, M., Vishkayi, S.I., Soleimani H.R.: The effect of geometric arrangement on the thermoelectric properties of Phenanthrene coupled to the graphene nanoribbons electrodes. Chinese J. Phys. 56, 2580-2588 (2018)

51. Brandbyge, M., Mozos, J.L., Ordejón, P., Taylor, J., Stokbro, K.: Density-functional method for nonequilibrium electron transport. Phys. Rev. B 65, 165401 (2002)

52. Taylor, J., Guo, H., Wang, J.: Ab initio modeling of quantum transport properties of molecular electronic devices. Phys. Rev. B 63, 245407 (2001)

53. Büttiker, M., Imry, Y., Landauer, R., Pinhas, S.: Generalized manychannel conductance formula with application to small rings. Phys. Rev. B 31, 6207 (1985)

54. Li, Z., Qian, H., Wu, J., Gu, B.L., Duan, W.: Role of Symmetry in the Transport Properties of Graphene Nanoribbons under Bias. Phys. Rev. Lett. 100, 206802 (2008) 


\section{Supplementary Files}

This is a list of supplementary files associated with this preprint. Click to download.

- SupportingInformation.tex 